\title{
Trapping of vibration energy into a set of resonators: Theory and application to aerospace structures
}

\author{
A. Carcaterra ${ }^{\mathrm{a}, *}$, A. Akay ${ }^{\mathrm{b}, \mathrm{c}}, \mathrm{C}$. Bernardini ${ }^{\mathrm{a}}$ \\ a Department of Mechanics and Aeronautics, University La Sapienza, Via Eudossiana, 18, Rome 00184, Italy \\ ${ }^{\mathrm{b}}$ Bilkent University, College of Engineering, 06800 Bilkent, Ankara, Turkey \\ c Carnegie Mellon University, Department of Mechanical Engineering, Pittsburgh, PA 15213, USA
}

\section{A R T I C L E I N F O}

\section{Article history:}

Received 5 December 2010

Received in revised form

9 April 2011

Accepted 11 May 2011

Available online 10 August 2011

Keywords:

Passive vibration damping

Apparent damping

Shock attenuation

Fuzzy damping

\begin{abstract}
A B S T R A C T
This paper presents the theory of a novel mechanism of energy absorption and induced damping in structural systems and its application to aerospace industry. The underlying principles of the physical phenomena have been addressed in several earlier publications, which focused on prototypical systems of absorbers that consist of a set of single-degree-of-freedom resonators. This paper generalizes those theoretical developments to the case of a cluster of beams attached to a continuous primary structure, to develop predictive methods for the expected performance of this new type of absorber, with particular emphasis on its optimal design. An embodiment of the conceived device is illustrated for an aerospace structure, a satellite, with the purpose of reducing the vibration of the electronic components on board during lift-off. Experimental results illustrate the feasibility and the attractiveness of this new absorption technique.
\end{abstract}

(c) 2011 Elsevier Ltd. All rights reserved.

\section{Introduction}

The effect of complex attachments on the dynamic response of built-up structures and their use as vibration absorbers have been the subject of numerous investigations in recent years. These studies examined different aspects of energy exchange between a so-called master structure that in the simplest case represented as a single-degree-of-freedom oscillator and a set of linear oscillators attached to it [1-15]. Several investigations (viz., [1-4]) showed that the numerous oscillators attached to a master structure collectively act like a viscous damper and increase its effective damping significantly. These results further suggested that damping induced by the attached oscillators on the master structure does not depend on the details of loss factor in each oscillator and that such a set of oscillators absorbs energy even for vanishing values of loss factor in each oscillator so long as the number of oscillators remain large, approaching infinity [1].

Weaver in a series of investigations [6-10] generally corroborated these results, providing alternative approaches and results, and validated the expression developed by Pierce et al. [1] for vanishing loss factors in the oscillators for early times, pointing out that, in the case of a finite number of oscillators, the assertions for vanishing loss factors hold true only for transient exchange of energy. At later times, energy returns to the master [6], behaving as a series of exponentially decaying pulses in the impulse response [3].

This subtle but significant difference relates to the issues surrounding modal overlap and the conditions under which it is permissible to represent the summation in the equations of motion by an integration as discussed previously by, for

\footnotetext{
* Corresponding author. Tel.: +3906 44585794; fax: + 3906484854.

E-mail addresses: a.carcaterra@dma.ing.uniroma1.it (A. Carcaterra), a.akay@cmu.edu (A. Akay).
} 
example, [3,4,14]. At a more fundamental level, however, the issue centers around the definitions of "apparent damping" that a master experiences as energy flows to the attached oscillators vs. true dissipation that converts vibratory energy into thermal energy, through loss mechanisms in the attached oscillators. (The one exception that crosses over these two cases would be a nonlinear system, such as a large number of atoms in a solid, that undergoes oscillations, which themselves represent thermal energy or true dissipation [16].)

Consequently, as Maidanik [14] pointed out, the actual dissipation of energy in a linear system requires a physical loss mechanism for the entire system. For a large number of oscillators, loss factor in each can be small so long as their sum is finite [14]. On the other hand, if the attached oscillators have zero loss, even for very large number of oscillators, their presence offers an apparent damping source. Weaver [6] pointed out that apparent damping in linear systems is a transient event followed by a periodic return of energy with a return time that depends on the number of oscillators. For a very large number of oscillators, the transient event has a very long duration.

These studies have shown that achieving irreversible energy transfer in conservative linear systems requires infinite degrees of freedom or infinite number of attached oscillators. However, when their number is finite, the mechanism of energy trapping in the attachment becomes more complicated $[17,18]$. For practical cases where the primary structure has a finite number of oscillators attached to it, the concept of irreversibility applies only during a transient period described as the return time during which energy flows into the satellite oscillators before returning to the primary structure.

The conditions that influence the return time were investigated to obtain a lower bound for it by an analysis of the reaction force on a rigid base by a finite number of oscillators demonstrating the influence of natural frequency distribution [17]. Based on these findings, the concept of linear energy sink was developed by optimizing the natural frequency distribution of the attached oscillators to minimize the energy retained by the structure to which they are attached [19]. The concept of linear energy sink was demonstrated through simple experiments that demonstrated how attached oscillators with the optimum frequency distributions can collectively absorb and retain the vibratory energy from an impulsively excited structure [20]. Analyses inspired by probably density distributions showed the existence of a family of frequency distributions for attached oscillators that lead to near-irreversibility of vibratory energy transfer [18-21]. These studies demonstrated the key characteristic of the frequency distributions as having condensation points, which lead to decaying impulse response function even in lossless systems, a phenomenon that can be observed in preloaded plates on elastic foundations and shells [22]. The loss-free linear oscillators with the same frequency distributions showed comparable performance in energy absorption with the cases when the oscillators in the complex attachment had nonlinearities resulting from impacts among them or having parametric stiffness [23]. The near-irreversibility observed in the studies cited above has close connections with energy flow between mechanical resonators, energy equipartitioning and entropy [24-27].

It is worth noting that the concept of damping in lossless systems has also been explored in control systems (viz., [28-30]), and also used as a basis for macroscopic irreversibility in thermodynamics [31] and for energy interaction in complex hybrid systems [32]. The idea of using complex attachments can be realized using different physical configurations and with mechanical as well as a set of electrical resonators. An electrical analog of the systems reported in $[18,19,21]$ is described in [33] that is based on the use of piezoelectric coupling. Another physical configuration assumes the master is a floating body in waves subjected to shock excitations as described in [34,35] and in this case the attachment, as reported in [33], has the twofold effect of mitigating the floating platform response and that of producing energy.

Many of these studies focused on time-domain response of the system and considered impulse response and short-time behavior. Building on these findings, the present paper extends the concept of apparent damping to a cluster of parallel beams and develops design rules for its application to a class of systems to absorb vibratory energy from primary structure. In particular, design and construct of an absorber and its application to a satellite is described.

The theoretical part, developed in Sections 2 and 3, synthesizes two basic results developed in time and frequency domains, together with physical interpretation of the induced equivalent damping. These results are then used to develop design rules for an optimal device and applied to a satellite, as illustrated in Sections 4 and 5.

\section{Apparent damping effect of a cluster of beams}

The purpose of this section is to derive the coupled equations of motion of a system that consists of a primary structure and an attached cluster of beams. The cluster consists of a set of $N$ elastic beams, each clamped at its mid-point to the same rigid support $\mathrm{AB}$, which is attached at point $P$ to the primary elastic structure by the rigid connector QP, as illustrated in Fig. 1.

\subsection{The coupled equations of motion}

The governing equation of motion, for a continuous, linear undamped primary structure is described by

$$
L[w(\mathbf{x}, t)]+m_{M}^{\prime} \frac{\partial^{2} w(\mathbf{x}, t)}{\partial t^{2}}=R \delta\left(\mathbf{x}-\mathbf{x}_{P}\right)
$$

where $\delta$ is the Dirac's distribution, $L[], w(\mathbf{x}, t)$ and $m_{M}^{\prime}$ are the structural operator, the displacement and the mass density, respectively, of the structure. $\mathbf{x}$ represents the spatial variable along the primary structure. $R$ describes the reaction force by the cluster on the primary at $\mathbf{x}_{P}$, where it is attached to the master. 


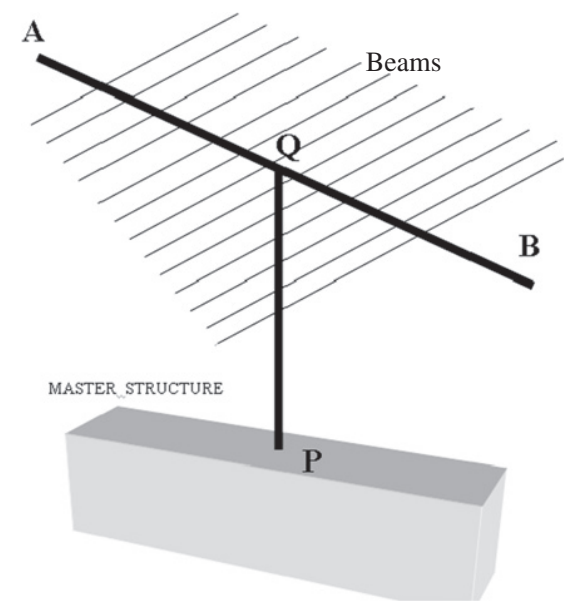

Fig. 1. Sketch of the master-cluster coupling.

Thus, the primary structure is coupled to the beams through $R$, which represents the sum of their clamping shear forces $T_{i}$ :

$$
R=\sum_{i=1}^{N} T_{i}=\left.\sum_{i=1}^{N} B_{i} \frac{\partial^{3} w_{i}}{\partial \xi_{i}^{3}}\right|_{\xi_{i}=0}
$$

where $B_{i}, w_{i}$ and $\xi_{i}$ represent the bending stiffness, flexural displacement (in the same direction as $w$ ) and the coordinate along the $i$ th beam of the cluster, respectively.

The $N+1$ coupled equations of motion are:

$$
\left\{\begin{array}{l}
L[w(\mathbf{x}, t)]+m_{M}^{\prime} \frac{\partial^{2} w(\mathbf{x}, t)}{\partial t^{2}}-\left.\delta\left(\mathbf{x}-\mathbf{X}_{P}\right) \sum_{i=1}^{N} B_{i} \frac{\partial^{3} w_{i}}{\partial \xi_{i}^{3}}\right|_{\xi_{i}=0}=0 \\
B_{i} \frac{\partial^{4} w_{i}\left(\xi_{\xi}, t\right)}{\partial \xi_{i}^{4}}+m_{i}^{\prime} \frac{\partial^{2} w_{i}\left(\xi_{i}, t\right)}{\partial t^{2}}=m_{i}^{\prime} \frac{\partial^{2} w\left(\mathbf{x}_{p}, t\right)}{\partial t^{2}}, \quad i=1,2, \ldots, N
\end{array}\right.
$$

where $m_{i}^{\prime}$ is the mass per unit length of the $i$ th beam. The second of Eq. (3) represents the motion of the elastic beams clamped at a moving base, the primary structure. The motion of the base AB provides a uniformly distributed inertial force $m_{i}^{\prime}\left(\partial^{2} w\left(\mathbf{x}_{P}, t\right) / \partial t^{2}\right)$. Representing with $\Phi_{i h}\left(\xi_{i}\right)$ and $q_{i h}(t)$, respectively, the $h$ th orthonormal mode of the clamped-free $i$ th beam and the associated principal co-ordinates of the primary, the beam vibrations within the cluster can be described by

$$
w_{i}\left(\xi_{i}, t\right)=\sum_{h=1}^{\infty} \Phi_{i h}\left(\xi_{i}\right) q_{i h}(t)
$$

where the modes are normalized with respect to mass density. Substituting these expressions in the second of Eq. (3) and using orthonormality conditions produces

$$
\ddot{q}_{i h}(t)+\omega_{i h}^{2} q_{i h}(t)=L_{i h} \frac{\partial^{2} w\left(\mathbf{x}_{p}, t\right)}{\partial t^{2}}
$$

where $\omega_{i h}$ is the $h$ th natural frequency of the $i$ th beam with

$$
L_{i h}=\int_{0}^{l_{i}} m_{i}^{\prime} \Phi_{i h}\left(\xi_{i}\right) d \xi_{i}
$$

Solutions to Eq. (5) can be expressed as a convolution:

$$
q_{i h}(t)=\left[\Gamma(t) \frac{L_{i h}}{\omega_{i h}} \sin \omega_{i h} t\right] * \frac{\partial^{2} w\left(\mathbf{x}_{P}, t\right)}{\partial t^{2}}
$$

with $\Gamma(t)$ as the Heaviside's distribution.

With Eqs. (4) and (7), Eq. (3) can be rewritten as an integro-differential equation in terms of the primary structure displacement $w$ :

$$
L[w(\mathbf{x}, t)]+m_{M}^{\prime} \frac{\partial^{2} w(\mathbf{x}, t)}{\partial t^{2}}-\delta\left(\mathbf{x}-\mathbf{x}_{P}\right)[S(t) \Gamma(t)] * \frac{\partial^{2} w\left(\mathbf{x}_{P}, t\right)}{\partial t^{2}}=0
$$


where

$$
S(t)=\sum_{h=1}^{\infty} \sum_{i=1}^{N} \frac{U_{i h}}{\omega_{i h}} \sin \omega_{i h} t, \quad U_{i h}=\left.B_{i} L_{i h} \frac{\partial^{3} \Phi_{i h}}{\partial \xi_{i}^{3}}\right|_{\xi_{i}=0}
$$

Expressions similar to the kernel $S(t) \Gamma(t)$ in Eq. (8) have been shown to have remarkable properties [18]. Of particular interest among these properties is the one that allows, under certain conditions, replacement of the summation $(i=1, N)$ in $S(t)$, by an integral, which helps demonstrate the damping effect the cluster produces on the master.

Considering that each beam has its own discrete set of natural frequencies, the integration process replaces the summation across $h$ th natural frequency of the $N$ beams in the cluster with a continuous distribution of frequencies with frequency spacing between them approaching zero. Introduction of a dummy continuous variable $\chi=i / N$ that varies between 0 and 1 to the set of resonators, with $\Delta \chi=1 / N$, illustrates the approximation of the summation in Eq. (9) by an integral $I(t)$ :

$$
S(t)=\sum_{h=1}^{\infty} N \sum_{i=1}^{N} \frac{U_{h}\left(\chi_{i}\right)}{\omega_{h}\left(\chi_{i}\right)} \sin \omega_{h}\left(\chi_{i}\right) t \frac{1}{N} \approx I(t)=\sum_{h=1}^{\infty} N \int_{0}^{1} \frac{U_{h}(\chi)}{\omega_{h}(\chi)} \sin \omega_{h}(\chi) t d \chi
$$

where $\omega_{h}(\chi)$ represents the continuous distribution of the $h$ th natural frequency throughout the set of beams. The integral (10) can be evaluated by first replacing the integration variable $\chi$ by $\omega_{h}$ through $\left(d \omega_{h}(\chi) / d \chi\right)=f\left(\omega_{h}\right)$ and introducing new integration limits:

$$
I(t)=\sum_{h=1}^{\infty} N \int_{\omega_{h}(0)}^{\omega_{h}(1)} \frac{U_{h}(\chi)}{\omega_{h} f\left(\omega_{h}\right)} \sin \omega_{h} t d \omega_{h}
$$

where $f\left(\omega_{h}\right)$ represents the frequency density of the $h$ th mode of the beams. $U_{h}(\chi)$ in Eq. (11) can also be expressed in terms of $\omega_{h}$. For a set of clamped-free beams of different lengths and cross sectional areas, $U_{h}(\chi)$ can be expressed as

$$
U_{h}(\chi)=\frac{4 B(\chi) \beta_{h}^{2}}{l^{3}(\chi)}\left(\frac{\sinh \beta_{h}-\sin \beta_{h}}{\cos \beta_{h}-\cosh \beta_{h}}\right)^{2}
$$

where $\beta_{1}=1.875, \beta_{2}=4.694, \beta_{3}=7.85$, etc.

The distribution of natural frequencies $\omega_{h}(\chi)$ depends on the bending stiffness $B(\chi)$ and the length $l(\chi)$ distributions through $\omega_{h}(\chi)=\left(\beta_{h}^{2} / l^{2}(\chi)\right) \sqrt{\left(B(\chi) / m^{\prime}(\chi)\right)}$, where $m^{\prime}(\chi)$ represents mass per unit length for the beams in the set. Substitution of this last expression in Eq. (12) gives

$$
U_{h}(\chi)=\mu_{h} \omega_{h}^{2}(\chi) m(\chi), \quad \mu_{h}=\frac{4}{\beta_{h}^{2}}\left(\frac{\sinh \beta_{h}-\sin \beta_{h}}{\cos \beta_{h}-\cosh \beta_{h}}\right)^{2}
$$

where $m(\chi)=m^{\prime}(\chi) l(\chi)$ is the distribution of beam mass within the set, which can further be expressed in terms of frequency as $m\left(\omega_{h}\right)$, since $\omega=\omega_{h}(\chi)$.

Replacing $U_{h}(\chi)=\mu_{h} \omega_{h}^{2}(\chi) m(\chi)$ in Eq. (11), yields an approximate expression for $S(t)$ as

$$
S(t) \approx I(t)=\sum_{h=1}^{\infty} N \int_{\omega_{h}(0)}^{\omega_{h}(1)} \mu_{h} \frac{\omega_{h} m\left(\omega_{h}\right)}{f\left(\omega_{h}\right)} \sin \omega_{h} t d \omega_{h}
$$

which when substituted in Eq. (8) produces the explicit equation of motion for the primary structure

$$
L[w(\mathbf{x}, t)]+m_{M}^{\prime} \frac{\partial^{2} w(\mathbf{x}, t)}{\partial t^{2}}-\delta\left(\mathbf{x}-\mathbf{x}_{P}\right)\left[\sum_{h=1}^{\infty} N \Gamma(t) \int_{\omega_{h}(0)}^{\omega_{h}(1)} \mu_{h} \frac{\omega_{h} m\left(\omega_{h}\right)}{f\left(\omega_{h}\right)} \sin \omega_{h} t d \omega_{h}\right] * \frac{\partial^{2} w\left(\mathbf{x}_{P}, t\right)}{\partial t^{2}}=0
$$

Eq. (14) represents the main result of this paper, describing the dynamics of the primary structure in time domain. The third term in Eq. (14) represents the global action of the cluster on the primary explicitly in terms of the natural frequency distribution of the beams in the attached absorber. The next section examines the same relationship in the frequency domain to provide a clear picture of how a cluster of beams acts as a vibration absorber and a source of apparent damping.

\subsection{Equivalent damping of the cluster}

Through Fourier transform $F$, the frequency domain counterpart of the equation of motion (14) results

$$
L[W(\mathbf{x}, \Omega)]-m_{M}^{\prime} \Omega^{2} W(\mathbf{x}, \Omega)-\delta\left(\mathbf{x}-\mathbf{x}_{P}\right) F\left\{\left[\sum_{h=1}^{\infty} N \Gamma(t) \int_{\omega_{h}(0)}^{\omega_{h}(1)} \mu_{h} \frac{\omega_{h} m\left(\omega_{h}\right)}{f\left(\omega_{h}\right)} \sin \omega_{h} t d \omega_{h}\right] * \frac{\partial^{2} w\left(\mathbf{x}_{P}, t\right)}{\partial t^{2}}\right\}=0
$$

where the third term has a nonzero imaginary part, indicating an equivalent dissipation effect by the cluster that has particular frequency properties. 
The third term can be expanded and expressed as a convolution:

$$
-\Omega^{2} N W\left(\mathbf{x}_{P}, \Omega\right) \sum_{h=1}^{\infty} F\{\Gamma(t)\} * F\left\{\int_{\omega_{h}(0)}^{\omega_{h}(1)} \mu_{h} \frac{\omega_{h} m\left(\omega_{h}\right)}{f\left(\omega_{h}\right)} \sin \omega_{h} t d \omega_{h}\right\}
$$

where the Fourier transform $F\{\cdot\}=\int_{-\infty}^{+\infty}(\cdot) e^{-j \Omega t} d t$ of each are:

$$
F\{\Gamma\}=\pi \delta(\Omega)+\frac{1}{j \Omega}
$$

and

$$
\begin{gathered}
F\left\{\int_{\omega_{h}(0)}^{\omega_{h}(1)} \mu_{h} \frac{\omega_{h} m\left(\omega_{h}\right)}{f\left(\omega_{h}\right)} \sin \omega_{h} t d \omega_{h}\right\}=\int_{\omega_{h}(0)}^{\omega_{h}(1)} \mu_{h} \frac{\omega_{h} m\left(\omega_{h}\right)}{f\left(\omega_{h}\right)} \int_{-\infty}^{+\infty} e^{-j \Omega t} \sin \omega_{h} t d t d \omega_{h} \\
=-j \pi \int_{\omega_{h}(0)}^{\omega_{h}(1)} \mu_{h} \frac{\omega_{h} m\left(\omega_{h}\right)}{f\left(\omega_{h}\right)}\left[\delta\left(\Omega+\omega_{h}\right)-\delta\left(\Omega-\omega_{h}\right)\right] d \omega_{h}=-j \pi\left[\mu_{h} \frac{\Omega m(\Omega)}{f(\Omega)}\right] \Pi_{h}(\Omega)
\end{gathered}
$$

with

$$
\Pi_{h}= \begin{cases}1 & \text { for } \Omega \in\left[\omega_{h}(0), \omega_{h}(1)\right] \\ 0 & \text { elsewhere }\end{cases}
$$

The convolution then follows as

$$
F\{\Gamma(t)\} * F\left\{\int_{\omega_{h}(0)}^{\omega_{h}(1)} \mu_{h} \frac{\omega_{h} m\left(\omega_{h}\right)}{f\left(\omega_{h}\right)} \sin \omega_{h} t d \omega_{h}\right\}=-j \Omega\left[\pi^{2} \mu_{h} \frac{m(\Omega)}{f(\Omega)}\right] \Pi_{h}(\Omega)-\int_{-\infty}^{+\infty} \pi \mu_{h} \frac{\Pi_{h}(\zeta) \zeta m(\zeta)}{f(\zeta)(\zeta-\Omega)} d \zeta
$$

The integral on the right-hand side above represents the kernel of the Hilbert transform $H$, which can be evaluated as

$$
\int_{-\infty}^{+\infty} \pi \mu_{h} \frac{\Pi_{h}(\zeta) \zeta m(\zeta)}{f(\zeta)(\zeta-\Omega)} d \zeta=\pi^{2} \mu_{h} H\left\{\frac{\Pi_{h}(\Omega) \Omega m(\Omega)}{f(\Omega)}\right\}
$$

Thus, in its final equivalent form, Eq. (15) becomes

$$
L[W(\mathbf{x}, \Omega)]-m_{M}^{\prime} \Omega^{2} W(\mathbf{x}, \Omega)-\delta\left(\mathbf{x}-\mathbf{x}_{P}\right) W(\mathbf{x}, \Omega) N\left(\sum_{h=1}^{\infty} j \Omega\left[\pi^{2} \mu_{h} \frac{\Omega^{2} m(\Omega)}{f(\Omega)}\right] \Pi_{h}(\Omega)+\pi^{2} \mu_{h} \Omega^{2} H\left\{\frac{\Pi_{h}(\Omega) \Omega m(\Omega)}{f(\Omega)}\right\}\right)=0
$$

Compared with, $\left.L[W(\mathbf{x}, \Omega)]-m_{M}^{\prime} \Omega^{2} W(\mathbf{x}, \Omega)\right)$, the real part of the series in the above equation can be neglected, leaving

$$
L[W(\mathbf{x}, \Omega)]-m_{M}^{\prime} \Omega^{2} W(\mathbf{x}, \Omega)-j \Omega\left[\pi^{2} N \frac{\Omega^{2} m(\Omega)}{f(\Omega)} \sum_{h=1}^{\infty} \mu_{h} \Pi_{h}(\Omega)\right] W(\mathbf{x}, \Omega) \delta\left(\mathbf{x}-\mathbf{x}_{P}\right)=0
$$

This expression has the familiar form of equation of motion in the frequency domain for a single-degree-of-freedom oscillator with a frequency-dependent equivalent damping:

$$
C_{e q}(\Omega)=\pi^{2} N \frac{\Omega^{2} m(\Omega)}{f(\Omega)} \sum_{h=1}^{\infty} \mu_{h} \Pi_{h}(\Omega)
$$

An alternative but still significant expression of this apparent damping effect can be developed by considering the mass of $d N$ beams as $d m=m(\Omega) N d \chi=m(\Omega) d N$ with a natural frequency within the bandwidth $[\Omega, \Omega+d \Omega]$ and substituting in Eq. (19):

$$
C_{e q}(\Omega)=\pi^{2} \Omega^{2} \frac{d m}{d \Omega} \sum_{h=1}^{\infty} \mu_{h} \Pi_{h}(\Omega)
$$

The notable aspect of expressions (19) and (20) is that the resulting equivalent damping develops even in the absence of any dissipation effect in the classical sense, thus forming the basis for the concept of apparent damping.

\section{Physical considerations and properties of $C_{e q}$}

\subsection{Physical interpretation of $C_{e q}$ in frequency and time domains}

In the absence of any energy dissipation within the entire system, presence of a damping-like term in the equation of motion for the primary structure amounts to energy transfer from the primary to the beams and is seen by the primary as energy loss. In this sense, the cluster performs as a vibration or shock absorber, as demonstrated with simpler systems in [20].

It is worth noting here that the efficacy of the cluster as a vibration absorber can be high for transient vibrations, which follows from the approximation introduced in Eq. (10) when replacing the summation $S(t)$ by the integral $I(t)$ in deriving $C_{e q}$. The impact of this approximation on the effectiveness of $C_{e q}$ can be better observed in the time domain. In general, $S(t)$ and $I(t)$ differ by a remainder term $\mathfrak{R}=I-S$. As shown in [17], $\mathfrak{R}$ is small at early times such that within a suitable time 
interval $\left[0, t^{*}\right], \Re$. Thus, for $t<t^{*}, S \approx I$, and $|\mathfrak{R}| \ll|S| \approx|I|$, and the approximation of $S$ by $I$ is acceptable and Eqs. (19) and (20) represent the energy transfer accurately.

Moreover, using the wave analogy illustrated in [17], a reasonable value for $t^{*}$ can be estimated by

$$
t^{*} \approx \frac{2 \pi N}{f\left(\Omega_{t u n}\right)}
$$

where $f\left(\Omega_{t u n}\right)$ represents a function of the frequency of the primary structure to which the set of beams is tuned. It follows that for $t \in\left[0, t^{*}\right], C_{e q}$ represents the apparent damping effect on the primary, but not for times $t>t^{*}$ since, in general, at times longer than $t^{*}$, the energy that migrated to the set of resonators rapidly returns to the primary structure as a result of recurrence. However, there are exceptions to the recurrence phenomenon commonly seen in energy transfer among nondissipative linear systems, as outlined in [18].

In some remarkable cases, the energy return phenomenon described above can be prevented. Consider the remainder term $\Re$ above which depends on the functions $\omega_{h}(\chi)$, which represent the frequency distribution of the attached oscillators. Earlier studies have shown that a special class of $\omega_{h}(\chi)$ can minimize a suitable averaged value of the remainder $\mathfrak{R}$, thus allowing approximation of the summation by an integral with a minimum error. In a sense, these "optimal" frequency distributions make $t^{*}$ so large that the energy return to the primary is not observable in practice. For practical purposes, a large $t^{*}$, in real structures, is substantially equivalent to an infinite $t^{*}$. Indeed, even the smallest dissipation inherent in any physical system will be sufficient to transform most of the vibration energy trapped in the cluster into heat, leaving, at most, a negligible fraction of the energy initially to return to the primary beyond $t=t^{*}$.

Also, the return time and apparent damping are related such that the faster the energy transfer from the master to the cluster, the longer the time $t^{*}$ it takes for any remaining energy to return to the primary [17]. Such a relationship is equivalent to higher values of $C_{e q}$, and can also be derived from Eqs. (19) and (21) to show that $C_{e q}\left(\Omega_{M}\right) \propto t^{*}$.

The next section provides details of an effective absorber design that acts as an apparent damper using the optimal frequency distributions described above with increased performances in terms of both $C_{e q}$ and $t^{*}$.

\subsection{Optimal frequency distributions and properties of $C_{e q}(\Omega)$}

Theoretically, the best frequency distribution $\omega_{h}(\chi)$ is found by solving for a functional minimization of the square average of the remainder $\mathfrak{R}^{2}=\int_{C} \mathfrak{R}^{2} P d C$, where $P$ and $C$ are suitable weighting function and integration domain, respectively, as shown in [18]. The resulting solutions belong to families of exponentials. Among them, a rather simple and effective distribution proposed in $[20]$ is

$$
\omega_{\text {opt }}(\chi)=\frac{\Delta W}{2}\left[\frac{2 \chi-1}{|2 \chi-1|} \frac{e^{\alpha|2 \chi-1|}-1}{e^{\alpha}-1}+1\right]+\omega_{\min }
$$

where $\chi \in[0,1], \Delta W=\omega_{\max }-\omega_{\min }$ is the absorber frequency bandwidth, with $\omega_{\max }, \omega_{\min }$ maximum and minimum frequencies within the cluster, respectively, and $\alpha$ is a parameter that modifies the shape of the frequency distribution. (Optimal value of $\alpha$ for best energy absorption is shown to be about 2.5 [20].) For the design of the absorber under consideration here, the first modes of the $N$ beams are considered for the optimal distribution across the set of beams $\omega_{1}(\chi)=\omega_{\text {opt }}(\chi)$, assuming that the first natural frequencies will dominate their response. The corresponding beam lengths within the cluster then follow as $l(\chi)=\left(\beta_{1} / \sqrt{\omega_{\text {opt }}(\chi)}\right) \sqrt[4]{\left(B / m^{\prime}\right)}$, when all the beams of the cluster have the same bending stiffness $B$ and same cross-sectional area (same thickness $h$, width $b$ and mass per unit length $m^{\prime}$ for manufacturing purposes) differing only in length. It follows then that the frequency distribution for the $h$ th mode of all the beams in the set can be represented as

$$
\omega_{h}(\chi)=\left(\frac{\beta_{h}}{\beta_{1}}\right)^{2} \omega_{\text {opt }}(\chi)
$$

Each of the frequency distributions represented by (22) shows a typical trend for a steep slope starting at $\chi=0$, a fairly flat branch around $\chi_{\text {tun }}=0.5$, and another steep slope as $\chi$ approaches 1 (see Fig. 2).

Considering the frequency density function $f,(1 / f)=\left(1 / d \omega_{h} / d \chi\right)$ is a Gaussian-like function with a sharp peak around $\chi_{t u n}$, to which corresponds the tuning frequency $\omega_{\text {tun } h}=\left(\beta_{h} / \beta_{1}\right)^{2}\left(\omega_{\max }+\omega_{\min } / 2\right)$. As seen in Fig. 3, the values around $\omega_{\text {tun } h}$, the natural frequencies of the distribution $\omega_{h}(\chi)$ condense.

Expressions (19) and (21) also provide an intuitive understanding of the same optimal characteristics through the factor $(1 / f)$, which produces large values for $t^{*}$ and $C_{e q}$ around the tuning frequency $\omega_{t u n} h$. In fact, the optimal distributions described in [18-22], exhibit a characteristic flat branch at the tuning frequency, implying $f\left(\Omega_{t u n}\right)$ vanishes and $t^{*}$ becomes infinite. In addition to the typical peaks of the equivalent damping related to $(1 / f)$, frequency-averaged damping can be estimated using Eq. (20).

\subsection{Frequency-averaged damping}

The frequency intervals $\left[\omega_{h}(0), \omega_{h}(1)\right]$ for neighboring modes may or may not partially overlap. In cases for non-overlapping modal frequency distributions, such that $\omega_{h}(1)<\omega_{h+1}(0)$ for any $h,\left(\beta_{h+1} / \beta_{h}\right)\left(l_{\min } / l_{\max }\right)>1$, where $l_{\min }$, 


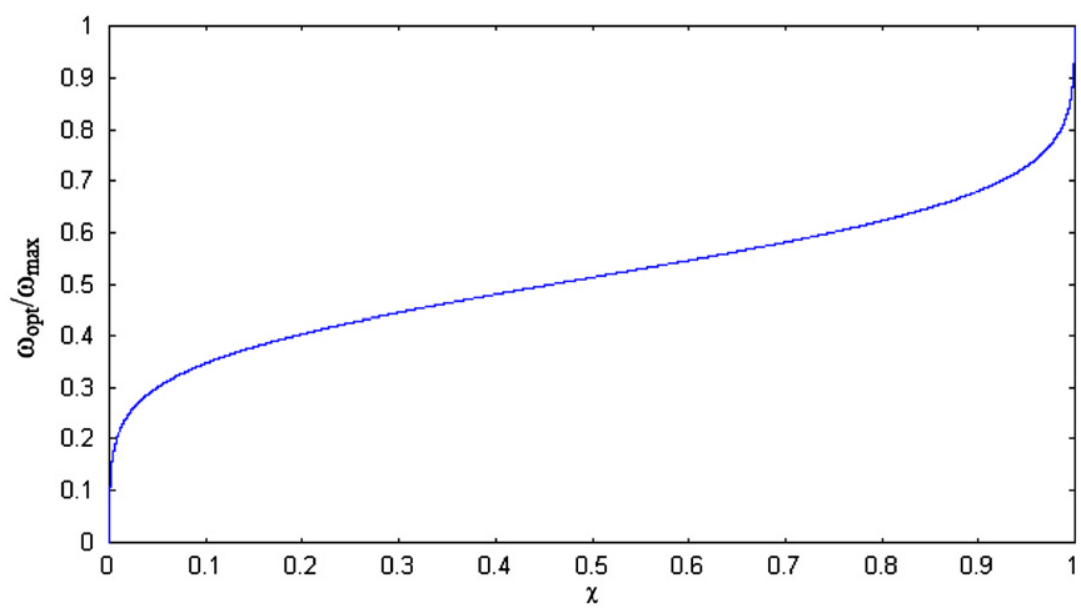

Fig. 2. Typical optimal frequency distribution within the cluster.

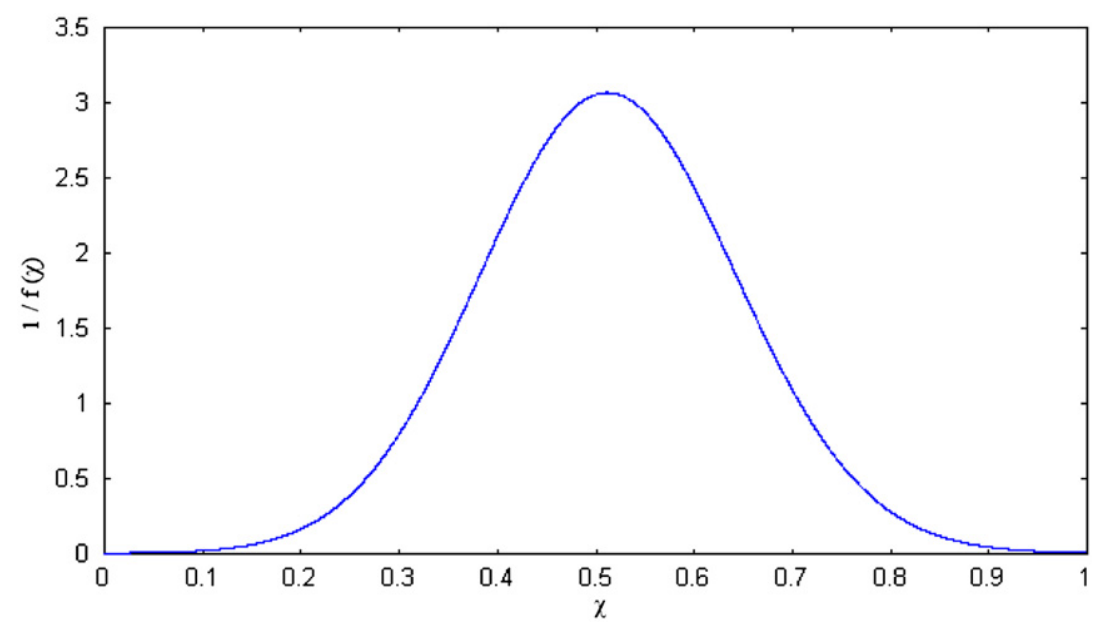

Fig. 3. Gauss-like frequency density distribution within the cluster.

$l_{\text {max }}$ represent the minimum and maximum beam lengths in the set. Then, in each frequency window $\Pi_{h}$, the damping expression simplifies as $C_{e q h}(\Omega)=\pi^{2} \Omega^{2}(d m / d \Omega) \mu_{h}$.

An estimate for the average of $C_{e q}$ over the frequency bandwidth $\Omega \in\left[\omega_{h}(0), \omega_{h}(1)\right]$ is expressed as

$$
\bar{C}_{e q h}=\frac{1}{\Delta W_{h}} \int_{\omega_{h}(0)}^{\omega_{h}(1)} \pi^{2} \Omega^{2} \frac{d m}{d \Omega} \mu_{h} d \Omega \approx \frac{\pi^{2} \omega_{t u n h}^{2}}{\Delta W_{h}} \mu_{h} M_{c l}
$$

where $\Delta W_{h}=\omega_{h}(1)-\omega_{h}(0)$ and $M_{c l}$ are the $h$ th natural frequency bandwidth of the device (absorber) and the total mass of the cluster, respectively.

In addition, the relationship between $M_{c l}$ and $N$ can be found as

$$
M_{c l}=m^{\prime} \sum_{i=1}^{N} l\left(\chi_{i}\right)=m^{\prime} N \sum_{i=1}^{N} l\left(\chi_{i}\right) \Delta \chi \approx m^{\prime} N \int_{0}^{1} l(\chi) d \chi=m^{\prime} N \beta_{1} \sqrt[4]{\frac{B}{m^{\prime}}} \int_{0}^{1} \frac{1}{\sqrt{\omega_{\mathrm{opt}}(\chi)}} \mathrm{d} \chi
$$

Eqs. (19)-(24) form the basis for an optimal design of the cluster, as shown in the next section.

\section{Cluster design and performances of the built-up device}

A device based on the theory described above was designed for use on board of UNISAT (UNIversity SATellite launched from Russia by a DNEPR rocket) (see Figs. 4-6), which is a permanent space project developed at the University of Rome La Sapienza by the Gauss Group. UNISAT is a small scientific satellite (14-20 kg depending on the payload), that has been launched periodically since 2000. The latest version of UNISAT will be equipped with the vibration suppressor described in this paper. Severe vibrations affect the electronic instrumentation of the satellite during lift-off of and the present device is 


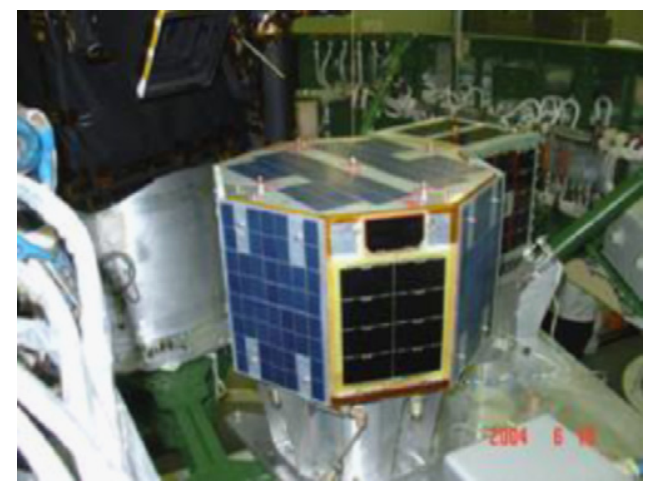

Fig. 4. UNISAT (version n. 3-launched in 2004). View of the satellites room before the launch.

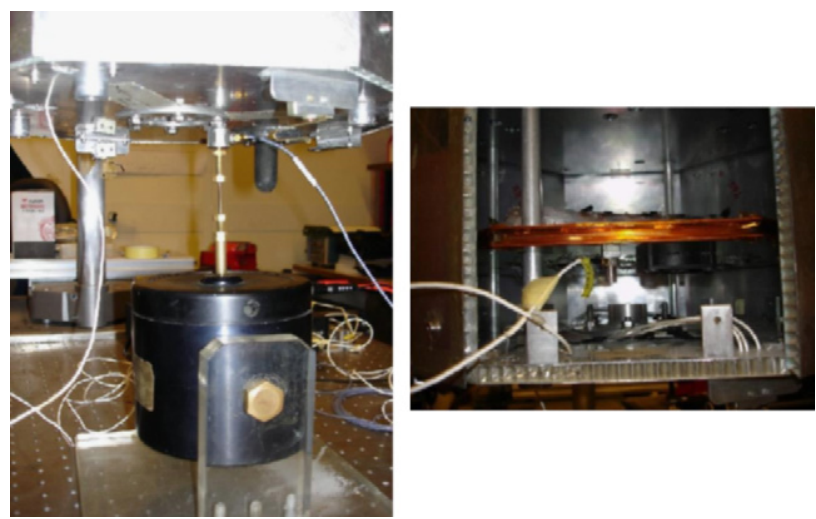

Fig. 5. On the left: electro-dynamic exciter acting on the bottom panel of the satellite. On the right: inner view of the satellite structure.

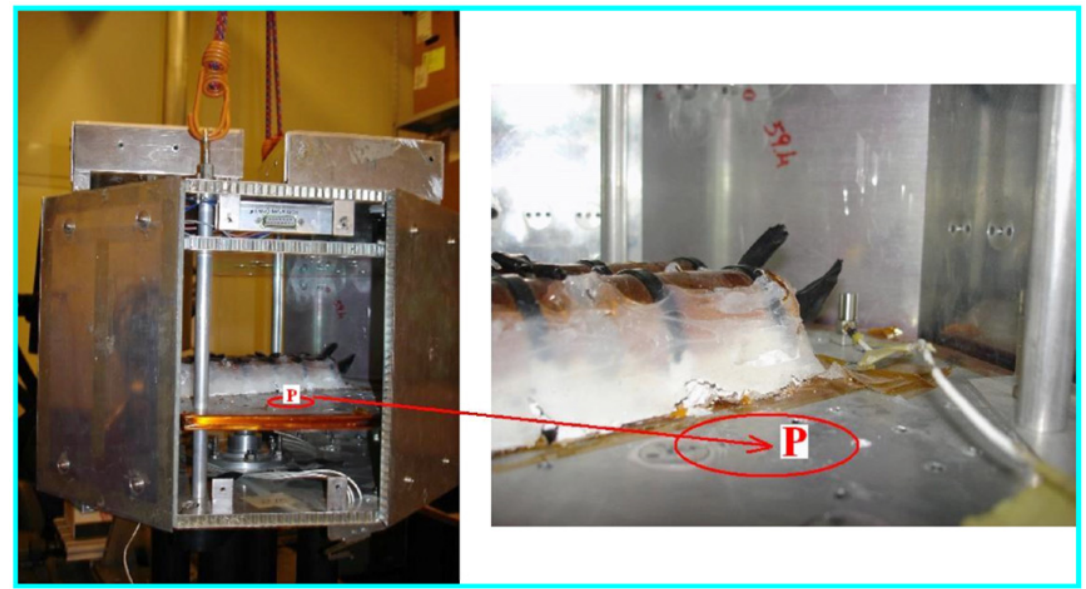

Fig. 6. Selection of the test "point $P$ " on board at which the suppression vibration device is applied.

designed to reduce the shock and vibration in the structure (one of the horizontal plates mounted inside the satellite) that carries the electronic package. Moreover, due to the safety requirements imposed, all electronic equipment on board the launcher must be switched-off during lift-off, making it unfeasible to use any active vibration suppressor.

The material used for the absorber is steel $\left(\rho=7780 \mathrm{~kg} / \mathrm{m}^{3}, E=187.5 \mathrm{GPa}\right)$ and the cluster of beams are manufactured by milling it from a stack of steel sheets, each with a thickness of $h=0.6 \mathrm{~mm}$. The maximum allowed space for the device is $90 \times 90 \times 40 \mathrm{~mm}$, with a maximum allowed mass of $0.15 \mathrm{~kg}$. 
The steps of the suggested design procedure are as follows:

(i) Selection of the frequency bandwidth and tuning frequency: On the basis of the experimental drive-point frequency response at the attachment point $P$ for $\Omega \in[0,1000 \mathrm{~Hz}]$, the frequency bandwidth $\Delta W$ and its central frequency $\omega_{\text {tun }}$ are selected as $\omega_{\text {tun }}=440 \mathrm{~Hz}, \Delta W=140 \mathrm{~Hz}$ based on the highest peak exhibited in the drive-point frequency response (see Figs. 6-7).

(ii) Optimal frequency distribution: The values for $\omega_{\max }, \omega_{\min }$ are determined from $\omega_{\text {tun }}=440 \mathrm{~Hz}, \Delta W=140 \mathrm{~Hz}$, which completely define the optimal frequency distributions given by Eq. (22), as represented in Figs. 8 and 9 .

(iii) Length distribution: The continuous length distribution is determined on the basis of $\omega_{\text {opt }}(\chi)$ from $l(\chi)=\left(\beta_{1} \sqrt{h} / \sqrt{\omega_{o p t}(\chi)}\right) \sqrt[4]{(E / 12 \rho)}$, which yields minimum and maximum lengths as 30.7 and $35.6 \mathrm{~mm}$, respectively. Considering that the device is made of symmetric beams (see Fig. 10), its maximum dimension, $81.2 \mathrm{~mm}$, is a sum of twice the length of the longest beam and the width of the rigid part $(10 \mathrm{~mm})$ to which all the beams are clamped, and is within the design constraint.

(iv) Number of beams: As indicated in Eq. (21), because the trapping time is proportional to the number of beams, $N$ can be selected to guarantee the energy stored within the cluster remains there long enough to be dissipated by the inherent internal damping. In order to have a large enough number of beams yet remain within the design constraints, the absorber design can be optimized such that only a small fraction $r$ of the energy initially trapped within the cluster can return to the primary. Vibration of each beam exhibits an energy decay associated with a harmonic motion at frequency $\omega$ expressed as $e^{-\eta \omega t}$, with $\eta$ representing the characteristic damping factor of the material. Since lower frequencies show slower decay, the lowest decay is associated with the lowest frequency $\omega_{\min }$ within the cluster. Using this property, the factor $e^{-\eta \omega_{\min } t_{d}}$ can be set equal to $r$ :

$$
e^{-\eta \omega_{\min } t_{d}}=r \rightarrow t_{d}=-\frac{\ln r}{\eta \omega_{\min }}
$$

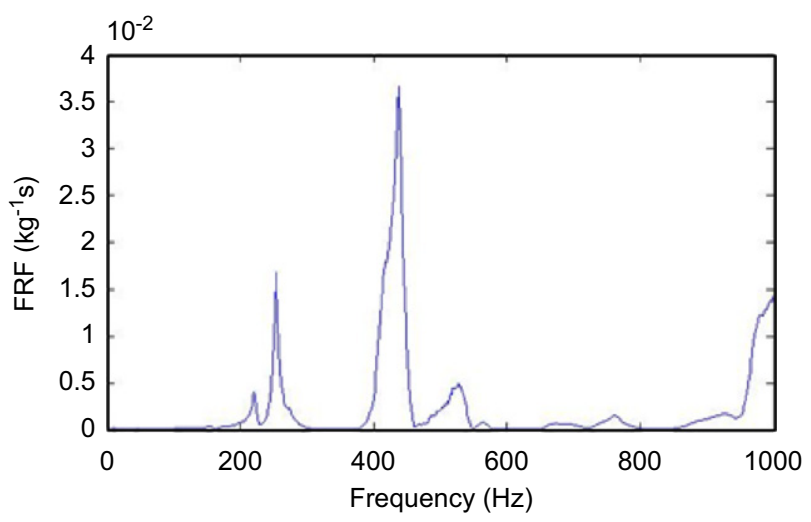

Fig. 7. Experimental response at the selected attachment point.

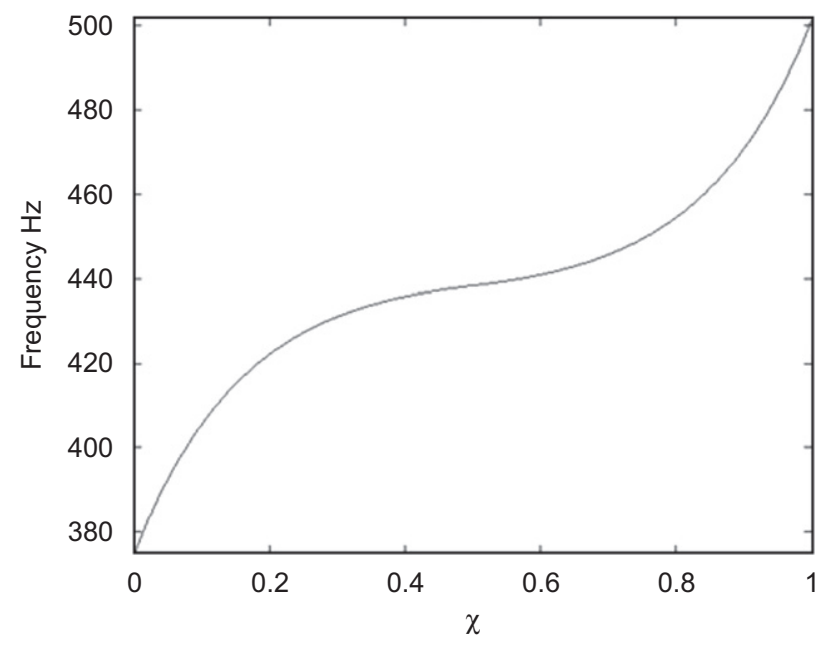

Fig. 8. Optimal frequency distribution. 


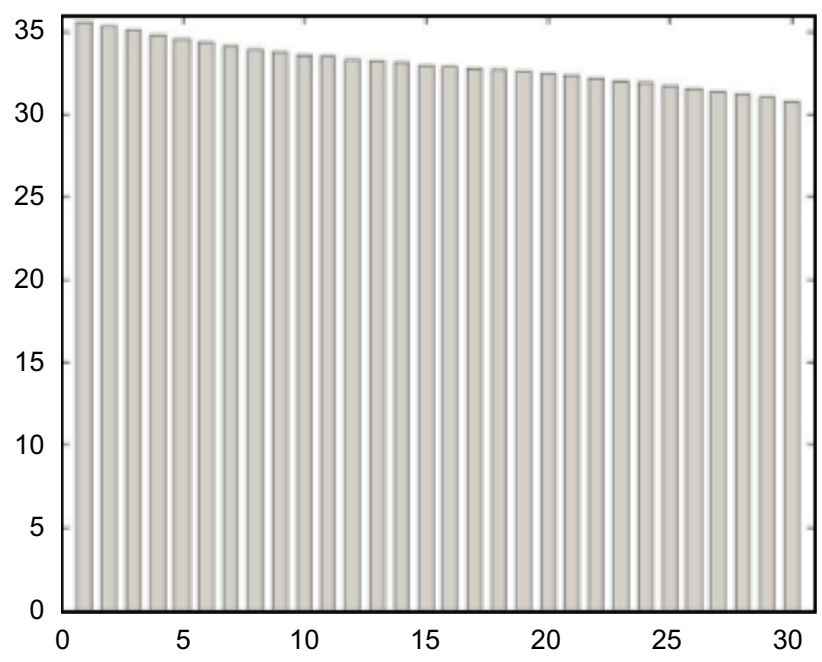

Fig. 9. Optimal length distribution within the set of beams of the device.
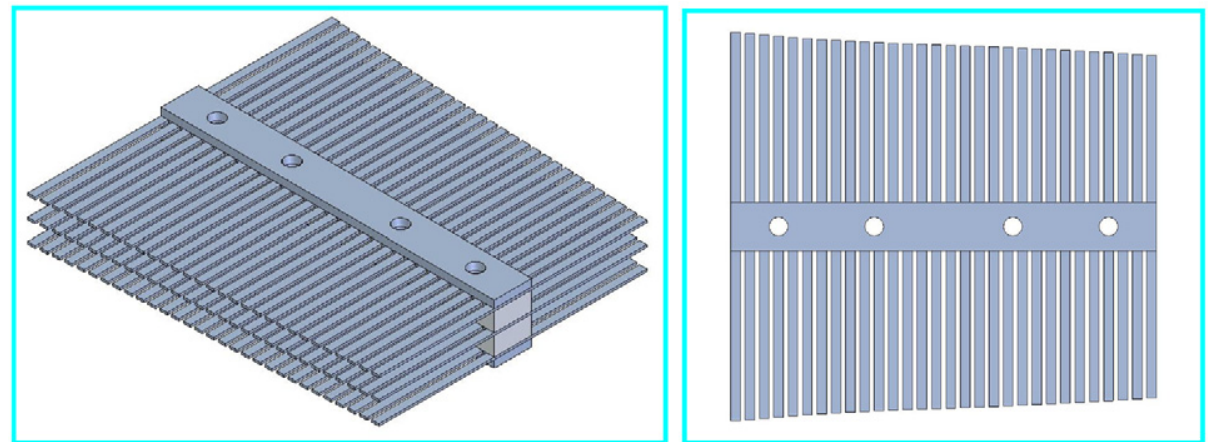

Fig. 10. Sketch of the design of the device.

Therefore for $t_{*} \approx t_{d}$ and substituting for the value of $t^{*}$ from Eq. (21) results in an expression for $N$ :

$$
N=-\frac{f\left(\omega_{t u n}\right) \ln r}{2 \pi \eta \omega_{\min }}
$$

that provides an order of magnitude for the number of beams needed for the cluster. Since $f\left(\omega_{\text {tun }}\right) \approx 500 \mathrm{rad} / \mathrm{s}, \omega_{\min } \approx 2350 \mathrm{rad} / s, \eta \approx 0.03$ and selecting a value for $r=0.01$, it follows that $N \approx 30$. For 30 beams, the corresponding discrete length distribution is represented in Fig. 9.

(v) Width and gap: Since the maximum allowed width of the device is also $D=90 \mathrm{~mm}$, sum of the beam widths, $b$, and the gaps, $g$, between adjacent beams, produces a relationship for the geometric configuration of the device as

$$
D=N b+(N-1) g
$$

Referring to expression (23), it is desirable within the design constraints to increase the total mass of the beams to increase the induced damping, which can be accomplished by maximizing the beam width $b$ and making $g$ as narrow as possible. In practice, however, the minimum gap size is determined by manufacturing limitations, which in this case was limited the value of $g=1 \mathrm{~mm}$. Then from previous Eq. (26), it follows that $b=2 \mathrm{~mm}$.

(vi) Maximizing the cluster mass: multiple layers: The previous analysis completely defines an optimal cluster satisfying the prescribed design requirements. Moreover, the height and weight of the beam-cluster module described above fell significantly below the prescribed constraints, leaving room for implementing multiple layers. A triple-layer absorber was constructed by stacking duplicates of the beam cluster within the maximum allowed height of $40 \mathrm{~mm}$. The stacked modules have twofold contributions to the overall damping. The increased additional mass, as suggested by Eq. (23) and the apparent damping through the additional clusters. The final device consists of three identical sets of beams stacked on top of each other, separated by two aluminum spacers. Each spacer has a height of $5 \mathrm{~mm}$, 
guaranteeing that the beams do not come into contact with those in adjacent clusters when undergoing the maximum accelerations during lift-off.

The device has been manufactured using an CNC milling machine that produced the set of beams a from a pack of aluminum sheets (see Fig. 11).

The final mass $M_{D E V}$ of the assembled device, shown in Fig. 12, weighs $0.13 \mathrm{~kg}$.

For the design values given above, the equivalent damping is estimated using Eq. (19).

Measurements to validate the estimated equivalent damping and the effectiveness of the absorber are performed following the procedure below.

As the first step, the best location for attachment point $P$ is identified considering both the response and the geometric constraints (Figs. 5 and 6). An electro-dynamic shaker was used to excite the structure with a spectrum similar to that under operating conditions while monitoring several sensitive positions of interest. The point where the maximum amplitude response develops is identified as $P$ (Fig. 6), selected among those where the installation of the device is

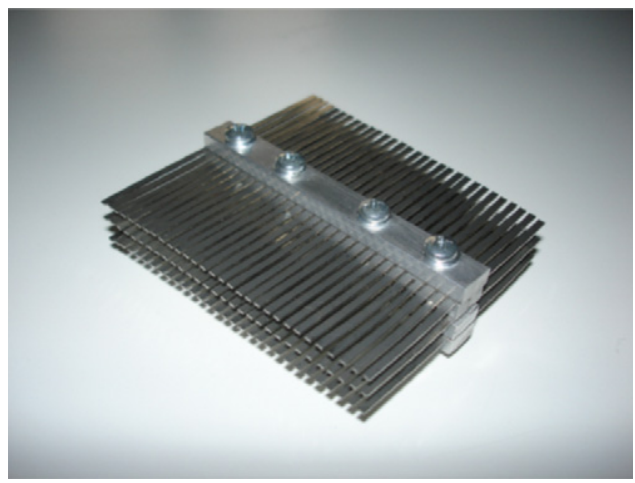

Fig. 11. View of the buildup device-tuning frequency $440 \mathrm{~Hz}$, total weight $130 \mathrm{~g}$.
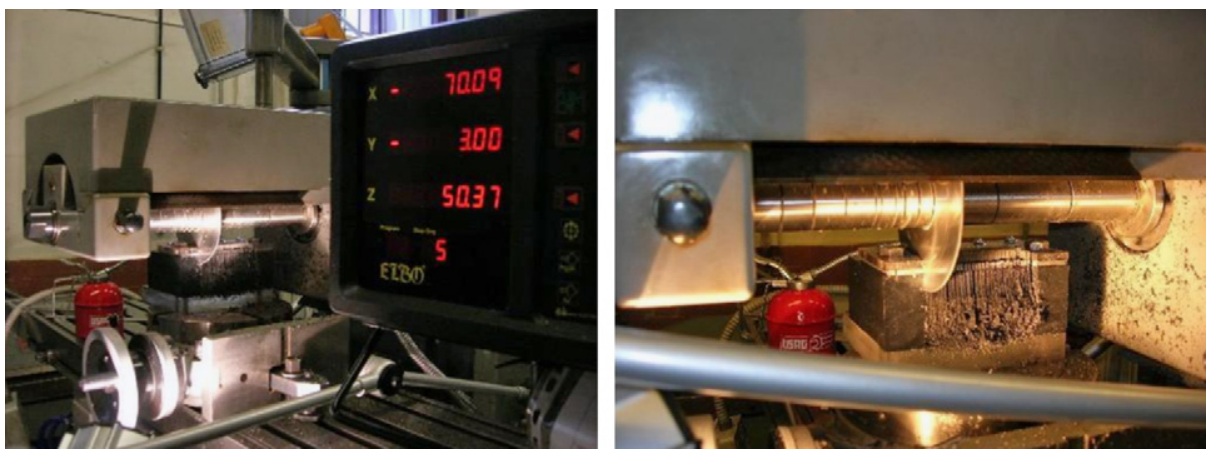

Fig. 12. Fabrication process of the device at the university lab by automated machining.

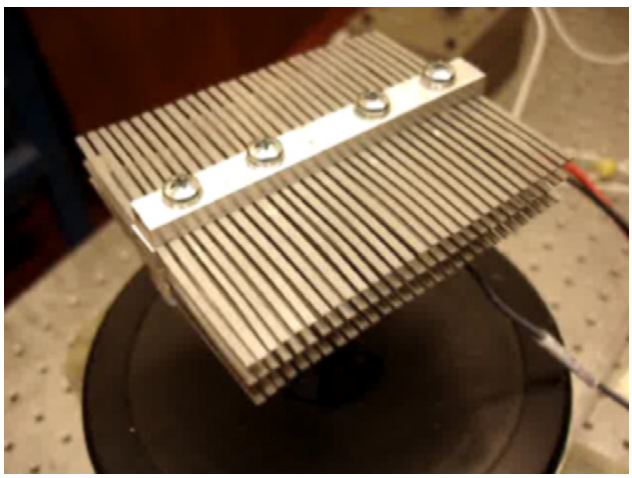

Fig. 13. Experimental test of the device alone to identify its apparent damping characteristics. 
technically possible. The peak frequency is identified from the drive-point frequency response function (FRF) at $P$ (Fig. 7 ) determining the tuning frequency and the bandwidth of the damper.

For the experimental identification of the apparent damping $C_{e q}^{E X P}(\Omega)$ of the device, first the experimental drive-point $\operatorname{FRF}^{E X P}(\Omega)$ of the device at the attachment point is measured (Fig. 13). The device is then attached to an electro-dynamic shaker and excited by a sweep-signal within the bandwidth $150-700 \mathrm{~Hz}$. The corresponding experimental apparent damping $C_{e q}^{E X P}(\Omega)$ is identified by curve fitting the measured FRF by a theoretical FRF of the form:

$$
\operatorname{FRF}^{E X P}(\Omega)=\frac{1}{-\Omega^{2} M_{D E V}+j \Omega C_{e q}^{E X P}(\Omega)}
$$

where $M_{D E V}$ is known $(0.13 \mathrm{~kg})$. The plot of $C_{e q}^{E X P}(\Omega)$ is shown in Fig. 14 and, as expected, the highest values appear in the vicinity of the tuning frequency of $438 \mathrm{~Hz}$. The measured peaks correspond to the 1st resonance frequency of each single

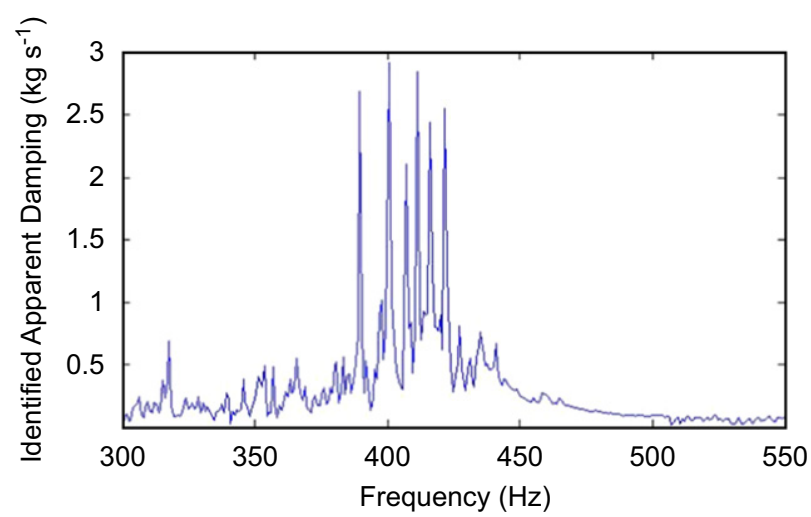

Fig. 14. Experimental measurement of the apparent damping of the device (tuning frequency $438 \mathrm{~Hz}$ ).
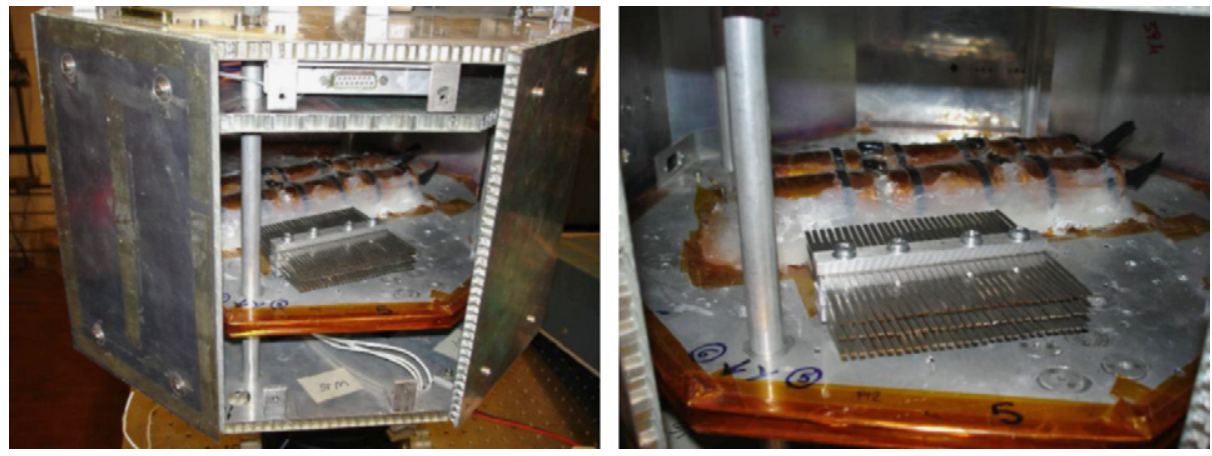

Fig. 15. View of the final installation of the vibration suppression device on board of UNISAT.

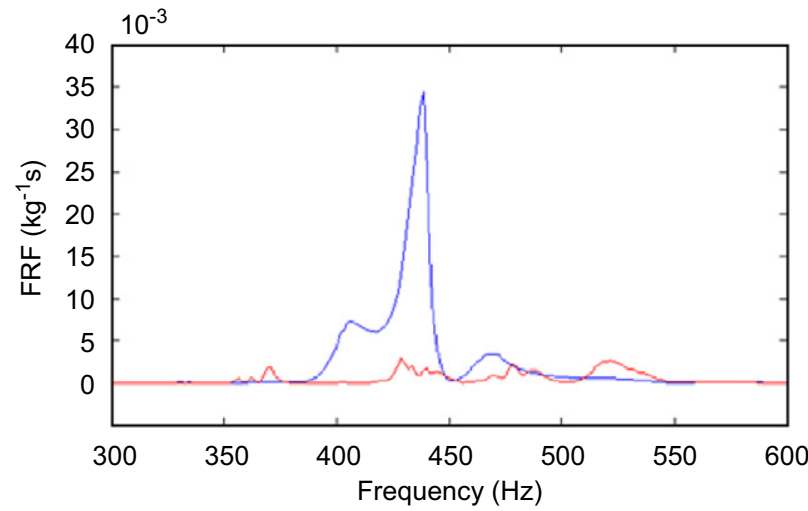

Fig. 16. Comparison of the FRFs at "point $P$ " with (red curve) and without (blue curve) the vibration suppression device. (For interpretation of the references to color in this figure legend, the reader is referred to the web version of this article.) 


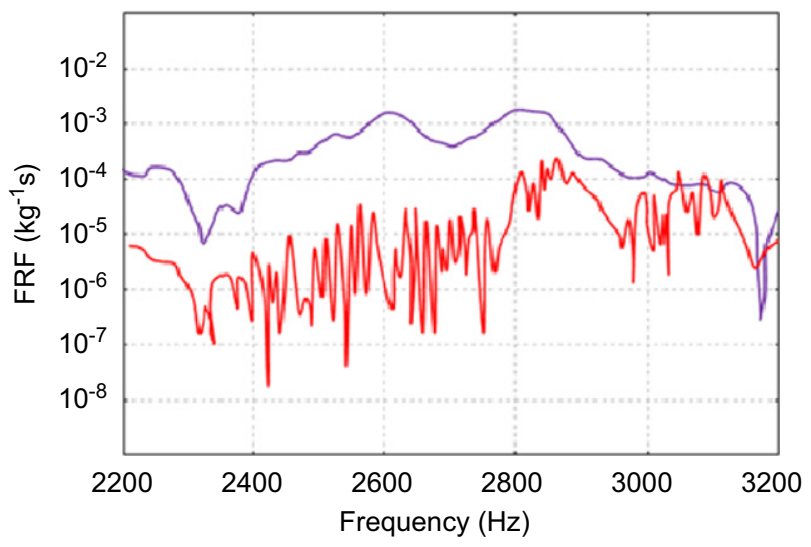

Fig. 17. Experimental evidence of the second modes effect on the suppression of vibration.

beam in the cluster. The multiple peaks in $C_{e q}^{E X P}(\Omega)$ is due to the presence of a finite number of beams in the set, which, in light of the previous theory, implies that the integral $I$ is replaced by the summation $S$.

To verify the effectiveness of the built-up device, it was installed on the satellite plate (see Fig. 15). Fig. 16 presents a comparison of the attenuated drive-point FRF with absorber mounted on it and one without, where the measurements refer to the point of attachment of the electronic package to the same plate at which the suppressor is installed. The results, given for the frequency range covered by the set of first modes of the beams in the cluster, show significant reduction in the FRF amplitude, and is expected to provide sufficient protection.

Fig. 17 shows the same comparison (log-scale on the vertical axis) but for the frequency bandwidth 2200-3000 Hz. This bandwidth covers the set of natural frequencies of the second modes of the beams producing, as explained for Eq. (19), an additional attenuation of the amplitude of vibration.

\section{Conclusions}

This paper presents a theory, followed by a set of design rules, for a new shock absorbing device that consists of a cluster of parallel resonating beams that absorb vibratory energy from a primary structure to which it is attached. The working principle of the shock absorber relies on a special distribution of beam lengths within the cluster such that energy flow from the primary structure to the device is maximized but return of energy to the primary is prevented. The paper also describes a prototype shock absorber built using the same design rules for application in a satellite. Measurements conducted on the prototype validate the estimated energy absorption and show effective reduction of vibration amplitude of the component to which it is attached.

\section{References}

[1] A.D. Pierce, V.W. Sparrow, D.A. Russel, Fundamental structural-acoustic idealization for structure with fuzzy internals, J. Vib. Acoust. 117 (1995) 339-348.

[2] M. Strasberg, D. Feit, Vibration damping of large structures induced by attached small resonant structures, J. Acoust. Soc. Am. 99 (1996) 335-344.

[3] M. Strasberg, Continuous structure as "fuzzy" substructures, J. Acoust. Soc. Am. 100 (1996) 3456-3459.

[4] K. Xu, T. Igusa, Dynamic characteristics of multiple substructures with closely spaced frequencies, Earthquake Eng. Struct. Dynam. 21 (1992) 1050-1070.

[5] R.J. Nagem, I. Veljkovic, G. Sandri, Vibration damping by a continuous distribution of undamped oscillators, J. Sound Vib. 207 (1997) $429-434$.

[6] R.L. Weaver, The effect of an undamped finite degree of freedom "fuzzy" substructure: numerical solutions and theoretical discussion, J. Acoust. Soc. Am. 100 (1996) 3159-3164.

[7] R.L. Weaver, Mean and mean-square response of a prototypical master/fuzzy structure, J. Acoust. Soc. Am. 101 (1997) $1441-1449$.

[8] R.L. Weaver, Multiple-scattering theory for mean response in a plate with sprung masses, J. Acoust. Soc. Am. 101 (1997).

[9] R.L. Weaver, Mean-square response in a plate with sprung masses, energy flow and diffusion, J. Acoust. Soc. Am. 103 (1998) $414-427$.

[10] R.L. Weaver, Equipartition and mean-square response in large undamped structures, J. Acoust. Soc. Am. 110 (2001) $894-903$.

[11] G. Maidanik, K.J. Becker, Noise control of a master harmonic oscillator coupled to a set of satellite harmonic oscillators, J. Acoust. Soc. Am. 104 (1998) 2628-2637.

[12] G. Maidanik, K.J. Becker, Characterization of multiple-sprung masses for wideband noise control, J. Acoust. Soc. Am. 106 (1999) $3109-3118$.

[13] G. Maidanik, K.J. Becker, Criteria for designing multiple-sprung mass for wideband noise control, J. Acoust. Soc. Am. 106 (1999) $3119-3127$.

[14] G. Maidanik, Induced damping by a nearly continuous distribution of nearly undamped oscillators: linear analysis, J. Sound Vib. 240 (2001) 717-731.

[15] J.F. Vignola, J.A. Judge, A.J. Kurdila, Shaping of a system's frequency response using an array of subordinate oscillators, J. Acoust. Soc. Am. 126 (2009) 129-139.

[16] C.E. Celik, A. Akay, Dissipation in solids: thermal oscillations of atoms, J. Acoust. Soc. Am. 108 (2000) 184-191.

[17] A. Carcaterra, A. Akay, Transient energy exchange between a primary structure and a set of oscillators: return time and apparent damping, J. Acoust. Soc. Am. 115 (2004) 683-696.

[18] A. Carcaterra, A. Akay, Theoretical foundation of apparent damping and energy irreversible energy exchange in linear conservative dynamical systems, J. Acoust. Soc. Am. 121 (2007) 1971-1982. 
[19] I. Murat Koç, A. Carcaterra, Zhaoshun Xu, Adnan Akay, Energy sinks: vibration absorption by an optimal set of undamped oscillators, J. Acoust. Soc. Am. 118 (2005) 3031-3042.

[20] A. Akay, Z. Xu, A. Carcaterra, I.M. Koc, Experiments on vibration absorption using energy sinks, J. Acoust. Soc. Am. 118 (2005) 3043-3049.

[21] A. Carcaterra, A. Akay, I.M. Koç, Near-irreversibility in a conservative linear structure with singularity points in its modal density, J. Acoust. Soc. Am. 119 (2006) 2141-2149.

[22] A. Carcaterra, A. Akay, F. Lenti, Pseudo-damping in undamped plates and shells, J. Acoust. Soc. Am. 122 (2007) $804-813$.

[23] N. Roveri, A. Carcaterra, A. Akay, Vibration absorption using non-dissipative complex attachments with impacts and parametric stiffness, J. Acoust. Soc. Am. 126 (2009) 2306-2314.

[24] A. Carcaterra, Ensemble energy average and energy flow relationships for nonstationary vibrating systems, J. Sound Vib. 288 (3) (2005) 751-790.

[25] N. Roveri, A. Carcaterra, A. Akay, Energy equipartition and frequency distribution in complex attachments, J. Acoust. Soc. Am. 126 (2009) 122-128.

[26] A. Carcaterra, An entropy formulation for the analysis of energy flow between mechanical resonators, Mech. Syst. Signal Process. 16 (2002) $905-920$.

[27] D.S. Bernstein, S.P. Bath, Energy equipartition and the emergence of damping in lossless systems, in: Proceedings of the IEEE Conference on Decision and Control, 2002, pp. 2913-2918.

[28] S. Vidoli, F. dell'Isola, Vibration control in plates by uniformly distributed PZT actuators interconnected via electric networks,, Eur. J. Mech. A 20 (2001) 435-456.

[29] U. Andreaus, F. dell'Isola, M. Porfiri, Piezoelectric passive distributed controllers for beam flexural vibrations, J. Vib. Control 10 (2004) 625.

[30] S. Vidoli, F. dell'Isola, Modal coupling in one-dimensional electromechanical structured continua, Acta Mech. 141 (2000) 37-50.

[31] W.M. Hassad, V. Chellaboina, S.G. Nersesov, Thermodynamics, Princeton University Press, 2005 Princeton Series in Applied Mathematics.

[32] W.M. Hassad, V. Chellaboina, S.G. Nersesov, Impulsive and Hybrid Dynamical Systems, Princeton University Press, 2006 Princeton Series in Applied Mathematics.

[33] A. Carcaterra, A. Akay, Patent W02006/103291, Vibration damping device, 2006.

[34] A. Iafrati, A. Carcaterra, A. Ciappi, E.F. Campana, Hydroelastic analysis of a simple oscillator impacting the free surface, J. Ship Res. 44 (2000) 278-289.

[35] A. Carcaterra, E. Ciappi, A. Iafrati, E.F. Campana, Shock spectral analysis of elastic systems impacting on the water surface, J. Sound Vib. 229 (2000) 579-605. 